\title{
The role of toll-like receptors in multifactorial mechanisms of early and late renal allotransplant injury, with a focus on the TLR4 receptor and mononuclear cells
}

\author{
Sławomir Cezary Zmonarski ${ }^{1, A, C, D}$, Mirosław Banasiki, $1, A, C, E$, Katarzyna Madziarska, ${ }^{1, B, C, E}$, \\ Oktawia Mazanowska ${ }^{2, \mathrm{~A}, \mathrm{E}, \mathrm{F}}$, Magdalena Krajewska ${ }^{1, \mathrm{~A}, \mathrm{E}, \mathrm{F}}$ \\ ${ }^{1}$ Department of Nephrology and Transplantation Medicine, Wroclaw Medical University, Poland \\ ${ }^{2}$ Faculty of Dentistry, Wroclaw Medical University, Poland \\ A - research concept and design; $\mathrm{B}$ - collection and/or assembly of data; $\mathrm{C}$ - data analysis and interpretation; \\ $D$ - writing the article; $E$ - critical revision of the article; $F$ - final approval of the article
}

Address for correspondence

Katarzyna Madziarska

E-mail:kmadziarska@wp.pl

\section{Funding sources}

This article has been written thanks to the support of the Scientific Program No. ST.C160.17.021

of the Wroclaw Medical University, Poland.

Conflict of interest

None declared

Received on March 5, 2018

Reviewed on May 13, 2018

Accepted on August 9, 2018

Published online on April 5, 2019

Cite as

Zmonarski SC, Banasik M, Madziarska K, Mazanowska 0, Krajewska M. The role of toll-like receptors in multifactorial mechanisms of early and late renal allotransplant injury, with a focus on the TLR4 receptor and mononuclear cells. Adv Clin Exp Med. 2019;28(7):981-987. doi:10.17219/ acem/94139

DOI

$10.17219 /$ acem/94139

Copyright

Copyright by Author(s)

This is an article distributed under the terms of the

Creative Commons Attribution Non-Commercial License

(http://creativecommons.org/licenses/by-nc-nd/4.0/)

\begin{abstract}
The innate immune system is activated before an adaptive immune response. An expression of a particular toll-like receptor (TLR) in a transplanted kidney depends on the localization of specific cells (e.g., endothelium, elements of the nephron structure), recent pathology and the time passed since transplantation. The TLR4 receptor is expressed on renal tubular epithelial (RTE) and endothelial cells, podocytes, blood and interstitial monocytes/macrophages, and dendritic cells. While circulating in blood, some monocytes are attracted and penetrate the transplanted organ, where they supplement the donor's resident macrophages. The intensity of migration depends on the local activation of inflammation in the graft and on the expression of specific receptors on kidney endothelial cells and monocytes/macrophages. The percentage of cells with shifted TLR4 expression usually increases in circulating monocytes. The TLR4 and the biochemical stimulation cascade derived from it in any type of cell, including monocytes, undergo multi-level regulation with feedback loops with other components of the primary system, and are also dependent on the action of immunosuppression. Toll-like receptor 4 senses stimuli that make monocytes contribute differently both to acute/chronic kidney injuries and to the development of tolerance. After kidney transplantation, TLR4 expression and related cytokine production capacity may vary depending on past diseases and oncoming problems. Since conventional immunosuppression does not prevent chronic allograft injury (CAI), peripheral blood monocytes and TLR4 constitute candidates for diagnostic and therapeutic targets. Considering the mutual communication among various elements of the primary immune system, future therapeutic intervention should be directed toward factors directly or indirectly regulating the expression or post-receptor signaling of the TLR4 receptor.
\end{abstract}

Key words: innate immunity, kidney transplant, toll-like receptor 4, monocyte, allograft injury 
Kidney transplantation is still the most promising therapeutic option for renal failure patients. Such unfavorable events as delayed graft function (DGF) or chronic allograft injury (CAI) limit kidney graft survival. Determining a set of easy-to-use immune markers for individual risk factors can help anticipate upcoming complications and extend median transplant survival. The best recognized sets of immune markers focus on elements of adaptive immunity: antibody- or T-cell mediated rejection. ${ }^{1-4}$ The innate system is activated before an adaptive response. Cellular components of the system consist of phagocytic cells (e.g., monocytes/macrophages), antigen-presenting cells (e.g., monocyte-derived dendritic cells), natural killer cells, a subset of $\mathrm{T}$ and $\mathrm{B}$ cells participating in innate immunity, as well as epithelial and endothelial cells (natural barriers synthesizing cytokines and chemokines, recognizing danger signals). ${ }^{5}$ Part of the activity of native immunity is based on pattern recognition receptors (PRRs), which are shared by various cellular compartments, and recognize pathogen-associated molecular patterns (PAMPs) and danger-associated molecular patterns (DAMPs) ${ }^{5,6}$ sensing a "harmed or damaged self". 6,7

Pattern recognition receptors can be divided either according to localization (into soluble, membrane-bound and intracellular) or according to their functional properties/ structural homology (into NOD-like receptors, C-type lectin receptors, retinoic acid-inducible gene-like receptors, and toll-like receptors (TLRs)). ${ }^{8-10}$ Activation of PRRs is usually induced by phagocytosis, inflammation and maturation of antigen-presenting cells (e.g., macrophages and dendritic cells). Phagocytosis (induced by the cluster of differentiation (CD36) scavenger receptor recognizing microbial diacylglycerides) is a necessary step for the activation of some other intracellular PRRs (e.g., TLRs). ${ }^{5}$ Toll-like receptors are shared in different proportions both by non-immune cells (NIC, e.g., renal epithelial and endothelial cells) and by immune cells. ${ }^{10}$ In the kidney, TLR1-4, TLR6 and TLR9 are widely expressed on renal tubular epithelial (RTE) cells. TLR2 and TLR4 are also found on endothelial cells and on podocytes., ${ }^{7,8}$ Within the immune system, TLRs are expressed by cells of both the adaptive and innate compartments. Cells of the innate part that express TLRs include granulocytes (Grs), monocytes/macrophages (Mcs/Mfs) and dendritic cells (DCs). ${ }^{6,7}$ Granulocytes, mastocytes and Mcs/Mfs are considered key players in early innate allo-immune response, especially in transplantation. ${ }^{11,12}$ Part of the peripheral blood monocyte (PBMC) population penetrates the tissues, ${ }^{6,13}$ where they undergo transformation into macrophages in ways that are specific to particular organs, e.g., Kupffer cells in the liver or mesangial cells in the kidney. Monocytes are characterized by the surface expression of CD14, CD11b as well as MAC1/3 and CD68. ${ }^{7}$ For clinical purposes, monocytes are divided into 2 classes: M1 and M2. ${ }^{14-16}$ The M1 class represents activated or pro-inflammatory macrophages and can be induced by interferon $\gamma$ (IFN $\gamma$ ) or lipopolysaccharide (LPS). Upon stimulation, they may change phenotype to DCs. ${ }^{14,16}$ The M2 class monocytes are wound-healing and pro-fibrotic. ${ }^{14,16}$ The M2 cells are believed to be hyporesponsive to most TLRs and complement-mediated signals. ${ }^{15}$ Renal epithelial cells are normally surrounded by macrophages and DCs, which create a dense network. Macrophages have diverse functions, including traditional inflammatory phagocytic function and trophic roles. They are believed to participate in the maintenance of tissue integrity. ${ }^{5}$ All immune cells, including Mcs/Mfs and DCs, have a higher expression of TLR4 than nonimmune cells. ${ }^{12}$

This article focuses on particular elements of the innate immune system: TLRs, especially TLR4, mononuclear $\mathrm{Mc} / \mathrm{Mf}$ cells and their leading role in renal transplant injury.

\section{General consequences of TLR4 stimulation}

Like endogenous TLR4 ligands, DAMPs activate monocytes/macrophages cells at high concentrations, which contrasts with activation produced by low concentrations of a conserved lipid A. ${ }^{17}$

Stimulatory ligands - high-mobility group box (HMGB) proteins, fibrinogen $(\mathrm{Fb})$ and uric acid - are among the most recognized DAMPs. ${ }^{18}$ HMGB1 is a ligand of TLR4 (i.e., of endothelial cells), which is passively released from injured renal cells. ${ }^{15,19,20}$ High local concentrations of HMGB1 can activate both TLR4 and TLR9. ${ }^{18} \mathrm{Fi}-$ brinogen also causes early activation of TLR4. In cultured podocytes, $\mathrm{Fb}$ induces a release of chemokines. ${ }^{21}$ Negative regulators of TLRs include splice variants of signaling molecules, soluble TLRs, cleavage enzymes, as well as receptors of the TLR/interleukin (IL)-1R family member TIR8, also known as single interleukin-1 receptor-1 (IL-1R1), SIGIRR. ${ }^{22}$

The main factors triggering TLR4 include upregulation of cytokines, chemokines or co-stimulatory molecules. ${ }^{23}$ The PAMP/DAMP recognition leading to activation of TLR4 is a sequential process, requiring the cooperation and aggregation of several accessory molecules. Lipopolysaccharide-induced TLR4 activation begins with the transfer of LPS monomers to LPS-binding protein (LBP) and subsequently to cluster of differentiation 14 (CD14, cell membrane-linked or soluble). Finally, the LPS+CD14 complex is transferred to myeloid differentiation factor 2 (MD2), which associates with TLR4. ${ }^{12,24}$ Ligand binding to MD2 favors the dimerization of 2 TLR4+MD2 complexes to the form of an activated heterodimer (LPS.MD2+TLR4) on the plasma membrane. ${ }^{12}$ The co-receptor CD14 is engaged in the initiation of endocytosis of (TLR4.MD2.LPS $)_{2}$ heterodimers. ${ }^{5,12,17,19}$ When TLRs form hetero- or homodimers (e.g., TLR2/TLR6, TLR4/TLR4), an increase in TLR sensing efficiency is gained and subsequent dimerization of the cytoplasmic toll/IL-1 receptor domain 
(TIR) activates intracellular signaling cascades. ${ }^{5,6,12,25}$ The TLR4 is also the only TLR that activates both the cytosolic adapter protein myeloid differentiation primary response 88 (MyD88)-dependent and MyD88-independent signaling cascade pathways. ${ }^{5,12,17,19}$ Activation of MyD88 to the NF-kB pathway (in cooperation with complement C3 and C5 factors) leads to the production of pro-inflammatory cytokines such as TNF- $\alpha$ and IL- 6 through the proinflammatory response. ${ }^{18}$ The MyD88-independent pathway triggers the activation of interferon regulatory factor 3 (IRF3), leading to the production of type I IFN. ${ }^{5,12,17,19}$ There is also crosstalk between TLR4 and TLR3. ${ }^{26}$ Due to the crosstalk, different ligands can utilize components of TLR-dependent pathways to various degrees. ${ }^{19}$ TLR4MyD88-NF- $\mathrm{B}$ signaling seems to influence TLR3 function, which explains the synergistic effect between TLR4 and TLR3 triggering in macrophages that has been observed in acute lung injury. ${ }^{26}$ As a result of TLR4 stimulation of the dependent biochemical cascades, the generation of various short-chain RNA molecules (miRNA) becomes activated. For example, miRNA-511 positively regulates TLR4 signaling, but miRNA-200c silences MyD88 and impairs the LPS-induced expression of pro-inflammatory cytokines like IL-6, CXCL9 and TNF- $\alpha .{ }^{27}$

The TLR4 senses stimuli which contribute differently to acute kidney injury (AKI) or to CAI, and also to the development of tolerance in either NIC or bone marrowderived cells (BMDC). ${ }^{23,28}$

\section{Innate immunity: from brain death to reperfusion injury}

In brain-dead donors (BDD), the kidney is exposed to an excess of reactive oxygen species (ROS) and DAMPs. ${ }^{29}$ Both contribute to maintaining the local inflammatory response, indirectly stimulate a local increase in E-selectin expression, intracellular adhesion molecule (ICAM)-1 and vascular adhesion molecule (VCAM)-1, and favor infiltration of the kidney by donor T cells and macrophages. ${ }^{18}$ The transfer of a kidney from the BDD to the recipient includes several unfavorable stages. Endothelial cells, especially RTE cells, residing in the oxygen-sensitive region of the outer stripe of the medulla, are highly susceptible to ischemia. They undergo swelling and upregulate adhesion molecules (P- and E-selectins and ICAM-1). ${ }^{18}$ After blood perfusion of the kidney is restored, humoral and cellular elements of innate/adaptive immunity flow in and out of the graft. ${ }^{30,31}$ An inevitable consequence of the transplant procedure is renal ischemic and reperfusion injury (IRI), ${ }^{5,30}$ which is known to amplify sterile inflammation ${ }^{8,31}$ and increases the general immunogenicity of the transplanted organ. ${ }^{32,33}$ The release of DAMPs and ROS induced during explantation, storage, surgery, and microbial contamination accelerates ${ }^{19,31}$ and continues the activation of innate immunity. ${ }^{7,18}$ Brain-dead donors also differ from living donors (LDs) in terms of DAMP generation, e.g., by more intensive release of HMGB1 by renal tubular cells ${ }^{6,25}$ and of IL1 $\beta$, TNF- $\alpha$, IL12, and IL6 by local monocytes/macrophages and mature dendritic cells (mDCs) located inside the kidney. ${ }^{7,15}$

Several factors may contribute to damage of endothelia, e.g., anti-human leukocyte antibodies (HLA), ${ }^{34}$ non-HLA antibodies ${ }^{4,35}$ or shear stress causing shedding of glycocalyces with a subsequent increase in permeability. ${ }^{8,31}$ Cellular stress induces an increase in complement activation with $\mathrm{C} 3 \mathrm{a}$ (and $\mathrm{C} 5 \mathrm{a}$ ) generation ${ }^{15}$ by resident cells and infiltrating leukocytes. ${ }^{7,30}$ Experimental local inhibition of complement formation reduces TLR4 gene expression, while experimental TLR4 blockage decreases C3 and B factor expression. ${ }^{30}$ Crosstalk between the complement system and TLRs has been well described. Signals mediated through decay accelerating factor (DAF) ${ }^{9}$ or through the C3a receptor $(\mathrm{C} 3 \mathrm{aR})$ and $\mathrm{C} 5 \mathrm{a}$ receptor $(\mathrm{C} 5 \mathrm{aR})$ expressed on macrophages and DCs are known to regulate the TLR4-mediated cascade. ${ }^{15,30}$ Mannose binding lectin (MBL) is also considered a putative ligand for TLR4. ${ }^{9}$ Activated complement further upregulates endothelial adhesion molecules with subsequent excessive accumulation of inflammatory cells. ${ }^{8,30}$

The TLR2 and TLR4 are at the center of these inflammatory actions of IRI..$^{9,18,19}$ In the kidney, the majority of the constitutive expression of TLR4 (and TLR2) is found in glomerular endothelial cells, in podocytes and RTE cells. ${ }^{25}$ During reperfusion, changes in TLR expression may occur non-concurrently on all kidney cell types. The expression is increased both on endothelial and RTE cells following injury. Endothelia of the vasa recta of the inner stripe of the outer medulla exhibited TLR4 upregulation at $4 \mathrm{~h}$ following reperfusion compared with $24 \mathrm{~h}$ for RTE cells. $^{8,25}$ After TLR4 stimulation, the main upregulated cytokines are IL- 6 , IL-1 $\beta$ and TNF- $\alpha$. This is accompanied by increased expression of macrophage inflammatory protein-2 (MIP-2) and monocyte chemoattractant protein-1 (MCP-1). Both are involved in the recruitment of trafficking Grs and monocytes. ${ }^{8,11,19}$

Following extravasation in response to TLR2, TLR4 and TLR9 stimulation, monocytes continue the release of pro-inflammatory cytokines and chemokines implicated in neutrophil recruitment. ${ }^{6,13}$ Neutrophils often cluster around graft-infiltrating monocytes. ${ }^{6}$ Monocytes recruited into inflamed tissue can differentiate into macrophages or myeloid dendritic cells (MDCs), depending on the local microenvironment. ${ }^{12}$ Under the influence of different regulatory factors (e.g., specific miRNA or TLR ligands), MDCs can stop their development at the stage of immature cells or progress to mature MDCs (mMDCs). ${ }^{27}$ In inflammatory surroundings, mMDCs express a high density of class II major histocompatibility complex (MHC) molecules and co-stimulatory receptors. They become potent inducers of type 1 alloimmunity after transplantation. ${ }^{736}$ In human MDCs, TLR4 stimulation increases their 
glucose consumption and lactate production (required for the secretion of cytokines) through higher expression of co-stimulatory molecule CD86 and ATP production. ${ }^{12}$ TLR4 stimulation of macrophages from human kidney grafts induces nitric oxide (NO) secretion and decreases the activity of arginase- $1 .{ }^{37} \mathrm{~A}$ suboptimal response (due to low expression) or low-level TLR4 stimulation prevent MDCs from maturing. Immature MDC (iMDCs) express low levels of MHC class II and co-stimulatory receptors. ${ }^{5}$ If iMDCs present alloantigens, they do not initiate T-cell responses, but favor the differentiation of regulatory $\mathrm{T}$ cells (the "tolerogenic" state). ${ }^{36}$ In monocytes, macrophages or MDCs, TLR4-induced production of inflammatory cytokines (e.g., by LPS) leads to negative feedback mechanisms switching on with a short delay, resulting in the downregulation of TLR4 receptors and signaling cascades (from minutes to hours). This makes monocytes and MDCs hyporesponsive for as long as several days, ${ }^{36}$ and may explain the low DGF rate in patients with recurrent Gram-negative infections. In cases of Gram-negative bacterial infection, this hyporesponsiveness is reversible through antibiotic therapy. ${ }^{5}$

It is still a matter of debate how much activated infiltrating leukocytes, as opposed to intrinsic renal cells, contribute to a high general level of TLR4 expression found in kidneys with IRI. Opinions range from equal contributions to a more significant role of TLR4 on renal cells. ${ }^{19,38}$ Neutrophils and PBMCs that have abundant TLR4 expression are readily attracted to inflammatory sites, ${ }^{7}$ so the proportion of cells with low TLR4 and MyD88 expression may increase in monocytes that remain in circulation. ${ }^{11}$ In clinical conditions, this phenomenon can be observed in kidney transplant recipients in whom the average TLR4 expression of PBMCs is low within the first $24 \mathrm{~h}$ after transplantation. These patients often have DGF, the duration of which is in proportion to the early decrease in TLR4. ${ }^{11,32}$ Data from one of our studies indicates that the low-level TLR4 expression of PBMCs may persist for a long time in patients with a history of DGF. ${ }^{32}$

\section{Acute kidney injury}

Before renal transplantation, candidates with an increased predisposition to acute kidney rejection may be singled out from patients with undisturbed adaptive resistance parameters. These candidates may have higher TLR4 expression on CD14 ${ }^{+}$PBMCs and a greater capacity to induce tumor necrosis factor $\alpha$ (TNF- $\alpha$ ) and IL-6 after LPS stimulation..$^{21,25,36} \mathrm{~A}$ timeline of PBMC TLR4 expression including an acute allograft rejection in early post-transplant follow-up follows a different pattern than the line of a non-rejecting patient. The "rejector's line" takes on a characteristic $U$ shape with initial progressive downregulation of TLR4 expression which then returns toward the baseline. The downregulation of TLR4 expression in rejecting patients is associated with reduced TLR4dependent production of TNF- $\alpha$ by stimulated PBMCs. Although it is not enough to conclude that excessive TLR4 signaling is an independent risk factor for acute rejection (AR), early signaling through TLR4 may represent a rate-limiting step in the rejection cascade. In contrast, the "non-rejector's" TLR4 expression timeline begins at a lower point than the $U$ curve and remains more or less flat. ${ }^{36}$ The long-term impact of AR on PBMC TLR4 expression does not seem to be permanent. In our study, we found no difference in long-term PBMC TLR2/TLR4 expression between DGF+AR(-) and DGF+AR(+). ${ }^{32}$ A steady-state suboptimal TLR4 response may protect the organ recipient from rejection by minimizing the signaling required for both dendritic cell maturation and subsequent T-cell activation. This may underlie the low incidence of rejection in some patients with either lower levels of TLR4 expression or reduced TLR4 ligand responsiveness before a transplant, as well as the link between TLR4 polymorphisms and organ rejection rates. ${ }^{36}$ The effects of known TLR polymorphisms (e.g., the TLR4/C14 complex) on acute kidney rejection rates are not uniform. Analyses of CD14 allele and genotype distribution indicate a lower incidence of $\mathrm{AR}$ in homozygous wild-type $\mathrm{CD} 14 * \mathrm{C} / \mathrm{C}$, but without statistical significance. ${ }^{38}$ Most TLR4 polymorphisms (e.g., A299G/T399I) observed in renal transplant recipients have been connected with a decrease in TLR4 expression in monocytes and neutrophils. The A299G/T399I polymorphism presents a lower risk of atherosclerosis and AR. ${ }^{6,19,24,36,39}$ The findings of studies concerning the influence of the A299G/T399I polymorphism on the infection rate are discrepant. Some of them have reported more frequent severe bacterial infections (e.g., urinary) and cytomegalovirus infections, ${ }^{24,39}$ but others have found no difference. ${ }^{38}$ Corticosteroid pulse therapy is also associated with a major decrease in TLR4 expression. ${ }^{38}$

Early AR after kidney transplantation can be expected when neither recovery nor rapid deterioration of renal function is observed. Both parenchymal cells and a wide array of immune cells (monocytes/macrophages, $\mathrm{T}$ and $B$ lymphocytes) are involved in AR, along with humoral factors. ${ }^{21}$ Vigorous co-activation of TLR4 and CD14 increases HLA molecules with co-stimulatory receptor expression on antigen-presenting cells and induces proinflammatory cytokine production, which may finally lead to an adaptive immune response..$^{38}$ It is believed that AR begins when mMDCs and macrophages effectively present the donor's antigens to the recipient's T cells and activate $\mathrm{T}$ cells, leading to their further differentiation. Mature MDCs support $\mathrm{T}$ helper type 1 development. ${ }^{40}$ The triggering mechanism is multifactorial. One of the proposed theories considers the delayed effects of IRI-dependent cellular stress, which induces the production of C3a (and C5a) and the release of DAMPs within the donor's organ. ${ }^{15}$ In allogenic (not syngenic) transplants, there is an upregulation of TLR4 receptor expression on tubular cells and within 
glomeruli - on intrinsic renal cells (e.g., mesangial cells, podocytes and vascular endothelial cells). ${ }^{21}$ One consequence of vascular endothelial activation inside the kidney is upregulation of cytokines (TNF- $\alpha$, MIP-2 and MCP-1) and adhesion molecules (leukocyte rolling is mediated by E-selectin, P-selectin and L-selectin). ${ }^{15}$ Experimental data show that Ly $6 C^{\text {low }}$ monocytes patrol along the endothelium and scavenge microparticles that are attached to the endothelial cells. ${ }^{13} \mathrm{Up}$ to $30 \mathrm{~min}$ after the initiation of TLR3/4-mediated vascular inflammation, there is a mobilization of neutrophils ${ }^{19}$ and shortly thereafter a change of patrolling Ly $6 \mathrm{C}^{\text {low }}$ monocytes occurs. Subsequent accumulation of Ly $6 \mathrm{C}^{\text {low }}$ monocytes to endothelia depends on the dominating stimulation of a particular TLR type. Agonists for TLR2 and TLR9 recruit crawling monocytes within $30 \mathrm{~min}$; agonists for TLR3 and TLR4 first induce the arrival of granulocytes, followed by an influx of patrolling monocytes (which takes up to $2.5 \mathrm{~h}$ ). ${ }^{7,13,19}$ Following the leukocyte rolling, firm adhesion is mediated by the binding of ICAM-1 on endothelia to lymphocyte function-associated antigen-1 (LFA-1 or CD11a/CD18) present on leukocytes. This facilitates diapedesis and trafficking into the renal interstitium. ${ }^{19}$ The proportion of Mcs (from a BDD) to Mfs (from the recipient) in the graft interstitium changes gradually after transplantation. In an experimental model of early post-transplant rejection, most $\mathrm{CD}_{11} \mathrm{~b}^{+}$cells and DCs expressed donor but not recipient MHC-2. Most DCs are formed from precursors that reside in the kidney, whereas few DCs are formed from recipient precursors recruited upon local upregulation of MIP-2 and MCP- $1 .^{22}$

TLR4 activation is only one element of a complex mechanism for stimulating the release of pro-inflammatory cytokines, e.g., IL-1. Generating of IL-1 $\beta$ by monocytes/macrophages requires the conversion of pro-IL-1 $\beta$ to the active form by caspase- 1 activation through an inflammasome complex (e.g., NLRP3). NLRP3 assembly requires coordinated stimulation provided by TLR4 and an increase in the concentration of a number of substances present in the cell microenvironment: extracellular glucose, hyaluronan, uric acid, monosodium urate crystals, and ATP. ${ }^{15}$ The cytokines released by Mcs/Mfs and MDCs also depend to a great extent on crosstalk between complement factors (C3aR and C5aR) and TLR4. C3a/C3aR engagement in human Mcs/Mfs increases the release of TLR4-mediated cytokines, leading to enhanced Th17 responses ${ }^{15}$ and has been found to play a role in both cellular and humoral rejection. ${ }^{18}$ In transplant biopsy specimens of kidneys with AR, TLR4 induction correlates with local chemokine synthesis (CCL2 and CCL20). ${ }^{21}$

The magnitude of TLR-stimulated inflammation (i.e., caused by monocytes/macrophages) depends on the feedback control mechanisms. The absence of a negative regulator of TLR responses, e.g., the inhibitory receptor toll IL-1R 8 (toll-IL-1R8), enhances IRI and DC maturation after kidney transplantation. ${ }^{6}$ Toll-IL-1R8 mediates the suppression of TLR/IL-1R signaling on epithelial cells, monocytes, DC precursors, and NK cells. Toll-IL-1R8 is expressed at the highest levels in the kidney. Experimental deletion of toll-IL-1R8 in kidney allografts favors maturation of resident DC precursors, expansion of allo-reactive $\mathrm{T}$ cells, as well as inhibition of Treg generation and AR to a greater degree than deletion of toll-IL-1R8 in the kidney recipient. ${ }^{22}$

\section{Chronic graft injuries}

Chronic allograft injury remains a great challenge for transplant clinicians. It is characterized by arteriopathy, peritubular capillaropathy, interstitial fibrosis, and tubular atrophy. ${ }^{25}$ The precise cause of CAI remains unclear, but appears to be related to pathological changes due to organ preservation, IRI prior to and after organ implantation, ${ }^{17}$ drug-related nephrotoxicity (e.g., calcineurin inhibitors), and chronic immune injury ${ }^{25}$ with, e.g., anti-endothelial cell antibodies, anti-angiotensin II type 1 receptor (anti-AT1R) antibodies, anti-endothelin receptor antibodies (anti-ETAR), ${ }^{4}$ non-HLA antibodies, ${ }^{4,35}$ or anti-HLA antibodies (in our observation, about $50 \%$ patients with anti-HLA antibodies return to dialysis). ${ }^{35}$ A transplanted kidney undergoing CAI releases DAMPs of varying intensity. Several molecules are implicated in this process, including fragments of nucleic acids, extracellular matrices (hyaluronic acid, fibronectin, heparin sulfate), HMGB1, fibrinogen, uric acid crystals, heat shock proteins, and fibrin. ${ }^{37,41}$ Time-varying chronic DAMP delivery may cause accumulation of more TLR4 mRNA in grafts displaying CAI lesions as well as in those with apparently normal histology. ${ }^{42}$ On the other hand, evidence for TLR/DAMP signaling as a mediator of CAI in humans is only indirect and associative. ${ }^{25}$

In CAI, monocytes are recruited from the blood into the transplanted kidney with an intensity depending on the time and type of graft pathology causing its malfunction. This means that there is a functional balance between 2 groups of cells: those passing through the endothelium and those remaining in the blood. ${ }^{32}$ Stressed renal epithelia, ${ }^{37}$ tissue-resident monocytes and monocytes infiltrating injured tissue are danger sentinels and respond quickly and with a low signal threshold to the presence of PAMP/DAMPs with the secretion of proinflammatory cytokines $^{7}$ and chemokines (e.g., MCP-1 or transforming growth factor (TGF)- $\beta$ ). ${ }^{37}$ Monocytes infiltrating the graft are considered a major cell-damaging component in $\mathrm{CAI},{ }^{37}$ linking chronic endothelial dysfunction with TLR4 expression. ${ }^{43}$ Maturation of resident DCs can be fostered by both HMGB1 and hyaluronan fragments via TLR2 and TLR4. ${ }^{25}$ Over time after transplantation, the microenvironment at tissue sites induces a phenotypic change of monocyte into more macrophage-like cells, producing less IL-1 $\beta$ and IL- 6 but sustaining low-grade chronic inflammatory 
processes, thus contributing to transplant vasculopathy and fibrosis. ${ }^{7}$ This inflammatory milieu guides proliferation and collagen synthesis by myofibroblasts and fibroblasts. In experiments, TLR deficiency reduces the number of tissue macrophages, DCs and primed T cells, leading to reduced cytokine release and stimulation of myofibroblasts, and reduced activity of infiltrating TLR-deficient fibroblasts. In this way, TLR inhibition can ameliorate fibrosis. ${ }^{15,37}$ These findings indicate the possible contribution of the TLR4-dependent signaling cascade of resident renal cells to the development of chronic injury. ${ }^{6,8,25}$ A study which focused on a comparison between circulating blood monocytes of patients with CAI (deteriorating renal grafts with chronic immune-mediated graft damage under standard immunosuppression) and patients with well-functioning grafts (in the absence of immunosuppression) found contrasting expression of MyD88 and TLR4 between the 2 groups. In the CAI group, the percentage of monocytes with raised TLR4/MyD88 expression was higher at both the transcriptional and protein levels. There was no difference in the absolute count of monocytes detectable in the blood. Thus, PBMC TLR4 expression patterns may give an idea of local graft TLR4 expression. ${ }^{25,41,42}$ Effective treatment of AR of a kidney graft in the past seems to have a negligible effect on TLR4 or MyD88 expression in both the kidney and PBMC population in the future. ${ }^{11,32}$

Graft recipients with stable renal function and with known TLR4 polymorphisms have a lower expression of TLR4 in monocytes and neutrophils. ${ }^{41}$ Loss-of-function TLR4 mutations (D299G and T399I) have beneficial impact on both acute rejection and atherosclerotic events for the possible price of higher susceptibility to post-transplant infections. ${ }^{25}$ D299G polymorphism may occur more frequently in patients surviving 15 years after transplantation, and TLR4T399I seems to be present only in those recipients who did not manifest CAI, although the difference was not statistically significant. ${ }^{19}$ Experimental "polymorphic" animals have been found to be protected from CAI, with declining effects downstream from TLR2/4 deficiency, through MyD88 deficiency, to TRIF deficiency. ${ }^{37}$ Eritoran is a structural analog of the lipid A portion of LPS. In some studies, it has proven to be an antagonist of the pathophysiological effects of LPS by blocking translocation of NF-kB and decreasing expression of inflammatory cytokines. ${ }^{17}$ However, contrary to expectations, clinical trials failed to demonstrate benefits from eritoran therapy. ${ }^{5}$

Almost all transplant patients receive immunosuppressive therapy. It has been found that cyclosporine A (CsA) influences TLR signaling. Cyclosporine A induces endoplasmic reticulum stress, which triggers unfolded protein response (UPR), which in turn activates c-Jun $\mathrm{N}$-terminal kinases (JNKs) in several cell types. In renal epithelial cells, CsA upregulates TLR4 expression without increasing TLR4 gene expression. Cyclosporine A causes nuclear-to-cytoplasmic translocation of HMGB1, and its nephrotoxicity depends on an intact TLR4 signaling pathway. In macrophages, UPR promotes inflammatory cytokines following TLR4 activation. ${ }^{20}$ In our study involving mycophenolate mofetil, in PBMC from patients between 1 and 6 months (but especially from 3 to 6 months) after transplantation, we found a negative correlation between doses of mycophenolate mofetil per kilogram of body weight and TLR4 expression. ${ }^{32}$

\section{Summary}

Expression of particular (TLRs in the kidney depends on the localization of specific cells (e.g., endothelium, elements of the nephron structure), recent pathology and time after transplantation. The TLR4 is widely expressed on RTE cells, endothelial cells and podocytes. It is also expressed on the majority of white blood cells involved in adaptive immunity (e.g., lymphocytes) and in native ones (monocytes/macrophages and dendritic cells). By remaining in a proportional numerical equilibrium with cells circulating in the blood, a portion of the monocyte population is attracted to and penetrates the tissues, where they supplement the donor's resident macrophage population. The percentage of cells with shifted TLR4 expression among circulating monocytes usually increases, sometimes becoming a mirror of intrarenal inflammation, especially in patients with a history of DGF. The TLR4 and its monocytes undergo multilevel crosstalk feedback control and immunosuppressive influences. It senses stimuli that make monocytes contribute differently both to acute/chronic kidney injury and to the development of tolerance. Following a kidney transplant, TLR4 expression and the associated cytokine production ability may be connected with different pathologies, depending on past disease and oncoming problems. Because conventional immunosuppression and diagnostics focusing on the adaptive immune response do not prevent chronic allograft damage, monocytes (PBMCs) and TLR4 are good candidates for the role of diagnostic and therapeutic targets. ${ }^{37}$ Considering the mutual communication of various elements of the primary immune system, future therapeutic intervention should be directed toward factors directly or indirectly regulating the expression or post-receptor signaling of the TLR4 receptor.

\section{ORCID iDs}

Sławomir Zmonarski (1) https://orcid.org/0000-0002-0764-4939 Mirosław Banasik (1) https://orcid.org/0000-0002-0588-1551 Katarzyna Madziarska (1) https://orcid.org/0000-0002-3624-3691 Oktawia Mazanowska (1) https://orcid.org/0000-0001-7669-7543 Magdalena Krajewska (ㄱ) https://orcid.org/0000-0002-2632-2409

\section{References}

1. Martin-Gandul C, Mueller NJ, Pascual M, Manuel O. The impact of infection on chronic allograft dysfunction and allograft survival after solid organ transplantation. Am J Transplant. 2015;15(12):3024-3040. 
2. Vondran FW, Timrott K, Kollrich S, et al. Pre-transplant immune state defined by serum markers and alloreactivity predicts acute rejection after living donor kidney transplantation. Clin Transplant. 2014;28(9): 968-979.

3. Banasik M, Boratyńska M, Kościelska-Kasprzak K, et al. Non-HLA antibodies: Angiotensin II type 1 receptor (anti-AT1R) and endothelin-1 type A receptor (anti-ETAR) are associated with renal allograft injury and graft loss. Transplant Proc. 2014;46(8):2618-2621.

4. Banasik M, Boratyńska M, Kościelska-Kasprzak K, et al. The impact of non-HLA antibodies directed against endothelin-1 type A receptors (ETAR) on early renal transplant outcomes. Transpl Immunol. 2014;30(1):24-29.

5. Hato T, Dagher PC. How the innate immune system senses trouble and causes trouble. Clin J Am Soc Nephrol. 2015;10(8):1459-1469.

6. Kreisel D, Goldstein DR. Innate immunity and organ transplantation: Focus on lung transplantation. Transplant Int. 2013;26(1):2-10.

7. Benichou G, Tonsho M, Tocco G, Nadazdin O, Madsen JC. Innate immunity and resistance to tolerogenesis in allotransplantation. Front Immunol. 2012;3:73.

8. Cheung KP, Kasimsetty SG, McKay DB. Innate immunity in donor procurement. Curr Opin Organ Transplant. 2013;18(2):154-160.

9. Farrar CA, Keogh B, McCormack W, et al. Inhibition of TLR2 promotes graft function in a murine model of renal transplant ischemia-reperfusion injury. FASEB J. 2012;26(2):799-807.

10. Zhou TB. Role of toll-like receptors gene polymorphism in renal transplantation. J Recept Signal Transduct Res. 2014;34(1):12-14.

11. Andrade-Oliveira V, Campos EF, Goncalves-Primo A, et al. TLR4 mRNA levels as tools to estimate risk for early post-transplantation kidney graft dysfunction. Transplantation. 2012;94(6):589-595.

12. Perrin-Cocon L, Aublin-Gex A, Sestito SE, et al. TLR4 antagonist FP7 inhibits LPS-induced cytokine production and glycolytic reprogramming in dendritic cells, and protects mice from lethal influenza infection. Sci Rep. 2017;7:40791.

13. Imhof BA, Jemelin S, Emre Y. Toll-like receptors elicit different recruitment kinetics of monocytes and neutrophils in mouse acute inflammation. Eur JImmunol. 2017;47(6):1002-1008.

14. Meng XM, Tang PM, Li J, Lan HY. Macrophage phenotype in kidney injury and repair. Kidney Dis (Basel). 2015;1(2):138-146.

15. Asgari E, Le Friec G, Yamamoto $H$, et al. C3a modulates IL-1beta secretion in human monocytes by regulating ATP efflux and subsequent NLRP3 inflammasome activation. Blood. 2013;122(20):3473-3481.

16. Olingy $C E$, San Emeterio CL, Ogle ME, et al. Non-classical monocytes are biased progenitors of wound healing macrophages during soft tissue injury. Sci Rep. 2017;7(1):447.

17. Liu M, Gu M, Xu D, Lv Q, Zhang W, Wu Y. Protective effects of Toll-like receptor 4 inhibitor eritoran on renal ischemia-reperfusion injury. Transplant Proc. 2010;42(5):1539-1544.

18. Fung $A$, Zhao $H$, Yang B, Lian Q, Ma D. Ischaemic and inflammatory injury in renal graft from brain death donation: An update review. J Anesth. 2016;30(2):307-316.

19. Zhao H, Perez JS, Lu K, George AJ, Ma D. Role of Toll-like receptor-4 in renal graft ischemia-reperfusion injury. Am J Physiol Renal Physiol. 2014;306(8):F801-811.

20. Gonzalez-Guerrero C, Cannata-Ortiz P, Guerri C, Egido J, Ortiz A, Ramos AM. TLR4-mediated inflammation is a key pathogenic event leading to kidney damage and fibrosis in cyclosporine nephrotoxicity. Arch Toxicol. 2017;91(4):1925-1939.

21. Bergler T, Hoffmann U, Bergler E, et al. Toll-like receptor 4 in experimental kidney transplantation: Early mediator of endogenous danger signals. Nephron Exp Nephrol. 2012;121(3-4):e59-70.

22. Noris M, Cassis P, Azzollini N, et al. The Toll-IL-1R member Tir8/SIGIRR negatively regulates adaptive immunity against kidney grafts. J/mmunol. 2009;183(7):4249-4260.

23. Wu H, Chadban SJ. Roles of Toll-like receptors in transplantation. Curr Opin Organ Transplant. 2014;19(1):1-7
24. Nogueira E, Salomao R, Brunialti MK, et al. Expression of TLR4 and -2 in peripheral mononuclear cells in renal transplant patients with TLR4 gene polymorphism. Int Immunopharmacol. 2010;10:1481-1485.

25. Leventhal JS, Schroppel B. Toll-like receptors in transplantation: Sensing and reacting to injury. Kidney Int. 2012;81(9):826-832.

26. Ding $X$, Jin S, Tong Y, et al. TLR4 signaling induces TLR3 up-regulation in alveolar macrophages during acute lung injury. Sci Rep. 2017;7: 34278.

27. Sarma NJ, Tiriveedhi V, Ramachandran S, Crippin J, Chapman W, Mohanakumar T. Modulation of immune responses following solid organ transplantation by microRNA. Exp Mol Pathol. 2012;93(3): 378-385.

28. Schinstock CA, Stegall M, Cosio F. New insights regarding chronic antibody-mediated rejection and its progression to transplant glomerulopathy. Curr Opin Nephrol Hypertens. 2014;23(6):611-618.

29. Eikmans $M$, de Canck I, van der Pol $P$, et al. The functional polymorphism Ala258Ser in the innate receptor gene ficolin-2 in the donor predicts improved renal transplant outcome. Transplantation. 2012; 94(5):478-485.

30. Damman J, Daha MR, van Son WJ, Leuvenink HG, Ploeg RJ, Seelen MA. Crosstalk between complement and Toll-like receptor activation in relation to donor brain death and renal ischemia-reperfusion injury. Am J Transplant. 2011;11(4):660-669.

31. Jane-Wit D, Fang C, Goldstein DR. Innate immune mechanisms in transplant allograft vasculopathy. Curr Opin Organ Transplant. 2016;21(3): 253-257.

32. Zmonarski SC, Koscielska-Kasprzak K, Banasik M, et al. Lowering of messenger ribonucleic acid Toll-like receptors 2-4,9 in peripheral blood mononuclear cells in kidney allograft recipients, relationships with immunosuppressive treatment, and delayed graft function occurrence. Transplant Proc. 2016;48(5):1519-1525.

33. Hosseinzadeh $M$, Nafar M, Ahmadpoor $P$, et al. Increased expression of Toll-like receptors 2 and 4 in renal transplant recipients that develop allograft dysfunction: A cohort study. Iran J Immunol. 2017;14(1): 24-34.

34. Banasik $M$, Jabłecki J, Boratyńska $M$, et al. Humoral immunity in hand transplantation: Anti-HLA and non-HLA response. Hum Immunol. 2014;75(8):859-862.

35. Banasik M, Kościelska-Kasprzak K, Myszka M, et al. A significant role for anti-human leukocyte antigen antibodies and antibody-mediated rejection in the biopsy-for-cause population. Transplant Proc. 2014;46(8):2613-2617.

36. Testro AG, Visvanathan K, Skinner N, et al. Acute allograft rejection in human liver transplant recipients is associated with signaling through toll-like receptor 4. J Gastroenterol Hepatol. 2011;26(1):155-163.

37. Wang S, Schmaderer C, Kiss E, et al. Recipient Toll-like receptors contribute to chronic graft dysfunction by both MyD88- and TRIF-dependent signaling. Dis Model Mech. 2010;3(1-2):92-103.

38. Krichen H, Gorgi Y, Dhaouadi T, et al. Toll-like receptor 4 and CD14 gene polymorphisms in Tunisian kidney transplantation. Transplant Proc. 2013;45(10):3472-3477.

39. Vandewalle A. Toll-like receptors and renal bacterial infections. Chang Gung Med J. 2008;31(6):525-537.

40. Kim TH, Jeong KH, Kim SK, et al. TLR9 gene polymorphism (rs187084, rs352140): Association with acute rejection and estimated glomerular filtration rate in renal transplant recipients. Int $\mathrm{J}$ Immunogenet. 2013;40(6):502-508.

41. Nogueira E, Ponciano VC, Naka EL, et al. Toll-like receptors-related genes in kidney transplant patients with chronic allograft nephropathy and acute rejection. Int Immunopharmacol. 2009;9(6):673-676.

42. Braudeau C, Ashton-Chess J, Giral M, et al. Contrasted blood and intragraft toll-like receptor $4 \mathrm{mRNA}$ profiles in operational tolerance versus chronic rejection in kidney transplant recipients. Transplantation. 2008;86(1):130-136.

43. Kwon J, Park J, Lee D, Kim YS, Jeong HJ. Toll-like receptor expression in patients with renal allograft dysfunction. Transplant Proc. 2008; 40(10):3479-3480. 